\title{
Analysis of Technological Evolution for TV Displays Using S-Curves
}

\author{
Chan-Woo Park1), Mit-Eum Lee2), Seong-Tae Lee3), Yong-Won Song4)
}

\begin{abstract}
This paper presents a study of the evolution of TV displays using an S-curve analysis. It provides an important analysis of how systems have changed from the past to the present, and how future systems can be predicted. The S-curve analysis shows that the main parameter of value (MPV) is important, which allows the curve to be drawn, and the position of the evolution to be seen. Therefore, the MPV of a system was set, and an S-curve analysis was conducted. The S-curves for cathode-ray tube (CRT), plasma display panel (PDP), liquid crystal display (LCD), organic light emitting diode (OLED), quantum dot light emitting diode (QLED), and micro light emitting diode (Micro LED) displays were drawn using a patent analysis, and their evolution positions on the S-curve were determined. To analyze the development trends of TV displays and predict future display technologies, an S-curve was drawn for different MPVs. The S-curve analysis showed that future TVs are expected to use Micro LED displays, which has passed its birth period and is now in the growth stage.
\end{abstract}

Keywords: S-curve, Patent, Main Parameter of Value (MPV), Micro LED, Evolution

\section{Introduction}

The fourth industrial revolution has been an important topic of discussion in the industry in recent years. It has been predicted to create revolutionary changes in the industry and labor market by combining artificial intelligence and robot technology with industrial technologies.

Received(May 19, 2020), Review Result(1st: July 9, 2020, 2nd: August 28, 2020), Accepted(September 25, 2020)

1) (Student) 15073 Dept. Nano \& Semiconductor Engineering, Korea Polytechnic Univ., Sangidaehak-ro, Siheung-si, Gyeonggi-do, Korea

email: cksdn548@naver.com

2) (Student) 115073 Dept. Nano \& Semiconductor Engineering, Korea Polytechnic Univ., Sangidaehak-ro, Siheung-si, Gyeonggi-do, Korea

email: faith0916@naver.com

3) (Student) 115073 Dept. Nano \& Semiconductor Engineering, Korea Polytechnic Univ., Sangidaehak-ro, Siheung-si, Gyeonggi-do, Korea

email: stgogo123@naver.com

4) (Professor, Corresponding Author) 15073 Dept. Nano \& Semiconductor Engineering, Korea Polytechnic Univ., Sangidaehak-ro, Siheung-si, Gyeonggi-do, Korea

email: ywsong@kpu.ac.kr 
Therefore, in this era of fast-paced digital revolution, it is extremely important to correctly establish and quickly implement a predictable and creative development strategy for future technologies with minimal time and cost. Various methods from the past have been developed and utilized in connection with future technology[1]. The Delphi method, a representative method most often used in this field, is a method for predicting the future by exchanging opinions among members to create new ideas. The scenario technique is a method for conceiving and evaluating multiple scenarios based on other prediction techniques that subjectively describe virtual situations in the future[2]. Such future prediction techniques analyze group opinions based on questionnaires and correct the errors through re-questioning; however, these techniques are difficult to apply in reality because the analysis results are often abstract and macroscopic. It is, therefore, necessary to develop a methodology of future prediction that is easy to apply to research sites, while overcoming abstraction and subjectivity through an objective analysis.

To address the aforementioned problem, this paper proposed a method that applies the technological evolution trend being studied in the Theory of Inventive Problem Solving (TRIZ) as a future technology prediction technique. TRIZ is a creative problem-solving methodology published in 1956 by the Russian scientist G. Altschuller, and it has been continued to be researched, improved, and developed by various experts to this day. Altschuller developed creative problem-solving methods such as those entailing a contradiction matrix and standard solutions through an analysis of more than 200,000 patents. During this process, Altschuller discovered that technical systems were being developed with certain patterns. He summarized such developments as nine general trends for technical systems[3]. Currently, more patterns have been discovered and studied by TRIZ experts. Research teams led by B. Zlotin, a leading researcher in this field, have developed a directed evolution (DE) methodology that predicts and leads future technology development from a technology and market perspective and is being used in actual research sites[4][5]. The DE methodology, like many future prediction methods, has a limitation in that it is impossible to exclude the subjectivity of the analyst, but it remains a methodology with high objectivity, being based on an objective pattern called "laws of technical system evolution." This study discussed a life cycle (S-curve) analysis method of a technological system that can explain the large framework of technological systems evolution based on the TRIZ theory and that predicts future trends. A practical method was developed through a systematic approach toward the meaning of the life cycle analysis method (S-curve) and graph drawing method. Through a patent analysis, a method to determine the S-curve position was systematized. Furthermore, a study was conducted for the case of a TV 
display for application in an actual system. Because TV displays have changed according to various patterns despite their short history, they are a suitable research material[6]. Through an S-curve analysis of TV displays, this study is aimed at identifying the position and direction of development of each display technology and predicting the direction of future TV display technologies by analyzing the function of the TV system that the technological evolution is based on.

\section{Research Methodology}

\subsection{Technology Development Stage Analysis Using S-Curve}

A technological system can be defined as a collection of elements that can be combined by a certain principle underlying some functions desired by humans, and technological development means improving and advancing a system such that it can effectively perform the functions to satisfy human requirements[3]. Therefore, the development of a core characteristic function among numerous functions in the system development is the most important index, and the variable for an evaluation of the core characteristic function is defined as the main parameter of value (MPV)[3]. A functional improvement of a system or product can be measured using the MPV. In general, the change in MPV over time can be expressed in the form of an S-curve, which is divided into four stages: birth, growth, maturity, and decline of the system, as defined in the product life cycle in the existing industry and management.

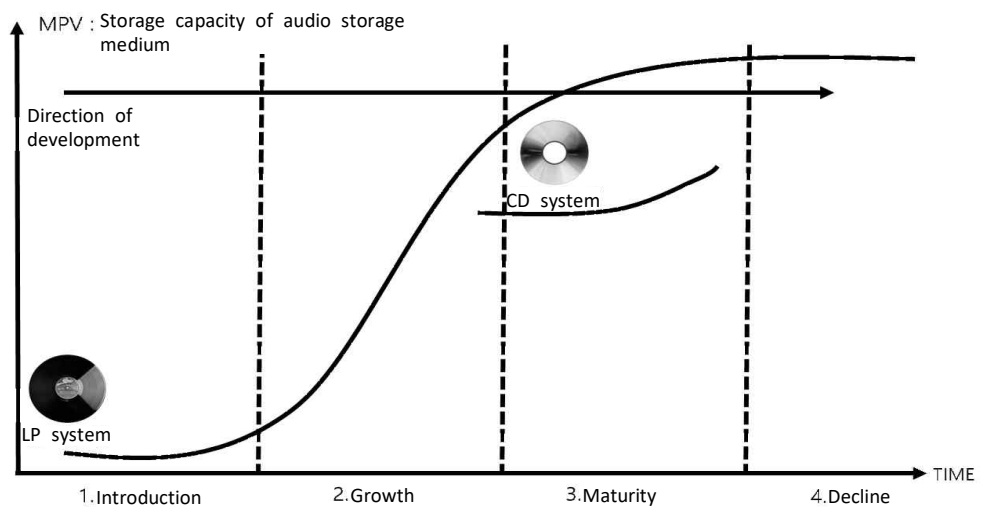

[Fig. 1] S-curve of Storage Capacity Evolution of Music Storage Media[7]

The main principles of a technological system are developed during the birth stage in the 
S-curve of the system, if the implementation principles of the core functions are previously developed. The growth stage occurs when the implementation of the functions rapidly increases, centering on the MPVs; the maturity stage occurs when a variety of product technologies are developed. In the decline stage, the growth of the product stops, and the product is eliminated from the market[3]. As shown in the S-curve of the audio storage medium in [Fig. 1], an LP plate (1931), the first audio storage medium, was introduced with the principle of listening to audio, which was information recorded based on the height of the groove on the plate, and played through the movement of the needle of the phonograph. The MPV of an information storage system is the storage capacity expressed as an S-curve that gradually increases with the $x$-axis (time) and is saturated. Although the storage capacity rapidly increased after the technological development of the grooves on the plate, the mechanical method for reading the signal by a needle in contact with such grooves hit a limit, and the technology showed no further improvement. With the advancement of new technology, a new idea called a compact disc (CD) (1982) for reading information using light was introduced to replace the LP plate method, and it started drawing its own S-curve. As such, an S-curve of a technological system shows the process of development from the birth of the system, based on a single core functional characteristic of the product, to its decline with the emergence of the next system[3].

\subsection{S-Curve Using Patent Analysis}

A system created based on a particular principle is developed through the stages of an S-curve: birth, growth, maturity, and decline, and it eventually disappears as it is replaced by another system created under a new principle[4]. A traditional method is to understand the four stages of an S-curve describing the development of technological systems and to identify and analyze where the current technological system is located through patents. The system can be judged by changes in the levels and number of patents.

[Fig. 2] shows the relationship between an S-curve and a patent in the introduction, growth, maturity, and decline stages through an analysis of patents related to the system, where the characteristics of the problems in each stage can be identified. A principle is used to create a function of a technological system in the birth stage, and the first system operated based on this principle is created. This is the time when the patent of the highest level is created. Although the level of this patent is the highest, the least number of patents are presented overall. The growth stage is the time when the system is improved through research and development. In the growth stage, the MPV increases rapidly, and the number of patents 
increases, although the level of patents continues to decline. In the maturity stage, it reaches a state in which the number of registered patents is significantly reduced because no more technological developments are made. Most of the difficult problems from the birth period have been resolved, and the level of patents continues to decline. As the maturity stage progresses, more studies are conducted aiming to reducing the costs than on developing technologies to enhance the performance of the product. During a state of decline, the number of patents stops increasing, and the product disappears into history with the introduction of a next-generation product that exceeds the efficiency of the existing system in the market[4]. Patents can be analyzed in various ways, and the representative method applied in South Korea is to use KIPRISS. It is possible to check what types of technical development trends exist and how they are evolving by synthesizing the number of patents registered to date through a KIPRISS patent analysis. A patent analysis demonstrates the process of how an old system disappears with the emergence of a new system. Therefore, the development process of an S-curve of a system can be identified by arranging the number of patents according to their timeline.

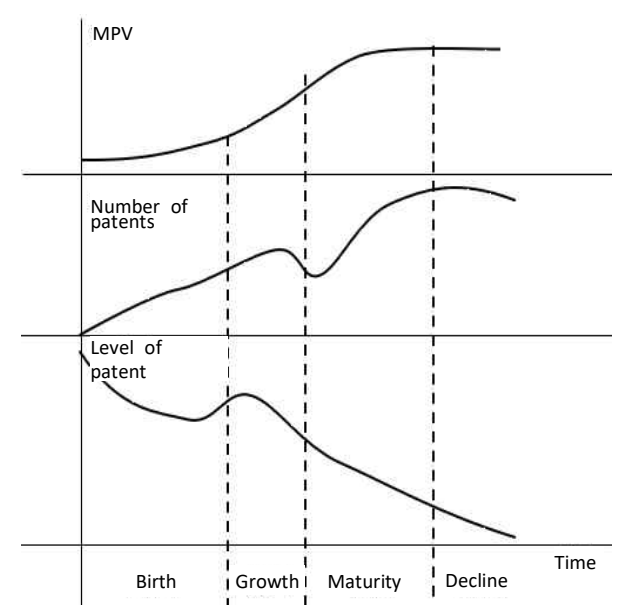

[Fig. 2] Relationship between S-curve and Patents of Birth, Growth, Maturity, and Decline Stages[3]

\subsection{Strategies for Each Stage of the S-Curve System Life Cycle}

Each system has an S-curve life cycle of "birth-growth-maturity-decline." Different strategies should be established depending on which stage of the S-curve life cycle the system is located. Therefore, it is necessary to determine where the system is located in the S-curve life cycle by analyzing the product-related patents or MPVs[3]. [Table 1] describes how to establish the strategies for the system in each stage[3]. 
[Table 1] Strategies for Each Stage of S-curve

\begin{tabular}{|l|l|l|l|l|}
\hline & Birth & Growth & Maturity & Decline \\
\hline $\begin{array}{l}\text { Char } \\
\text { acteri } \\
\text { stics }\end{array}$ & $\begin{array}{l}\bullet \text { The level of patents } \\
\text { is high, but the } \\
\text { number of patents is } \\
\text { small. }\end{array}$ & $\begin{array}{l}\text { MPV grows } \\
\text { rapidly. } \\
\bullet \text { Although the } \\
\text { number of patents } \\
\text { increases, the level of } \\
\text { patents decreases. }\end{array}$ & $\begin{array}{l}\bullet \text { MPV remains } \\
\text { stagnant. }\end{array}$ & $\begin{array}{l}\bullet \text { MPV starts } \\
\text { decreasing. }\end{array}$ \\
\hline $\begin{array}{l}\text { Strate } \\
\text { gies }\end{array}$ & $\begin{array}{l}\text { system to make the } \\
\text { most out of the } \\
\text { existing parts }\end{array}$ & $\begin{array}{l}\text { - Utilizing the } \\
\text { functions of the } \\
\text { system in new fields. }\end{array}$ & $\begin{array}{l}\text { - Combining with } \\
\text { other systems } \\
\text { - Developing a } \\
\text { next-generation } \\
\text { product }\end{array}$ & $\begin{array}{l}\text { Applying the } \\
\text { functions of the } \\
\text { system to new areas } \\
\bullet \text { Releasing a } \\
\text { next-generation } \\
\text { product }\end{array}$ \\
\hline
\end{tabular}

\section{S-Curve Analysis for Evolution of TV Displays}

\subsection{Main Parameter of Value (MPV) for TV Displays}

Defining the MPV of an S-curve requires an understanding of the functions of the technological system. A function of a technological system is defined as an action exerted to change or maintain the characteristics of the object of operation[5]. If this concept is applied to a TV display, it can be defined as a function to allow people to perceive the light within a specific bandwidth. MPVs can be derived through such definition of the function. The MPVs of a TV display that contribute to the function used to deliver light within a specific bandwidth to the human eye may include the viewing angle, resolution, image quality (PPI, aperture ratio, and luminance), and size of the display (in inches). MPVs can also be defined by observing them from the consumer side. All products are developed according to the needs of the consumers. The MPVs of a TV display derived according to the consumer needs may include the thickness of a TV, response time, and price. Consumers desire for thinner TVs to match the interior design of the homes, and an increasing number of consumers are demanding high response speeds with the development of more powerful gaming systems[6]. The price is the first concern of consumers when purchasing any product, not just TVs.

\subsection{Direction of Patent Analysis}

It is necessary to analyze the number of patents for an S-curve analysis. Analyzing the number of patents has an advantage in that the technological trend of a product can be identified, and 
a measure of technological change over time can be seen. Therefore, this study applied this concept to the display part, the most essential element of a TV, the technology of which has developed in various ways. To draw an S-curve, domestic patents listed on the KIPRISS patent website were analyzed. Because South Korean companies have been occupying the first and second places in the premium TV market, domestic patents could sufficiently reflect the trend of technological development[8]. The types of displays subject to the patent analysis included cathode-ray tubes (CRTs), plasma display panels (PDPs), liquid crystal displays (LCDs), organic light emitting diodes (OLEDs), quantum dot light emitting diodes (QLEDs), and micro light emitting diodes (Micro LEDs), and the number of recent patents and the cumulative number of patents were analyzed and shown as a graph. Based on the number of recent patents, the stages of birth and decline were identified, and the cumulative number of patents was used to determine the maturity stage. Each S-curve was analyzed using the number of patents.

\subsection{Results of TV Display Patent Analysis}

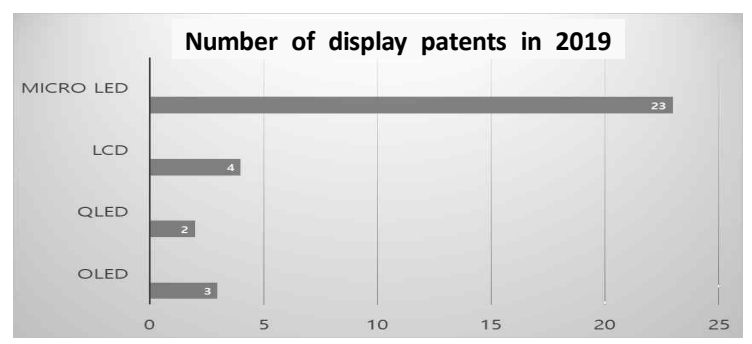

[Fig. 3] Number of Display Patents in 2019[9]

The number of patents in 2020 was excluded from the analysis because the year has not yet finished. The number of patents based on the most recent year, 2019, was analyzed. No TV-related CRT or PDP patents were registered, indicating that CRT and PDP technologies had faced their stage of decline. As of 2019, the number of patents registered for Micro LEDs was the largest, followed by LCDs, OLEDs, and QLEDs. [Fig. 3] shows that the number of Micro LED patents is far ahead of those of other displays, and the greatest numbers of studies are being conducted on this technology.

Considering the cumulative number of patents, it can be inferred that there are cases in which the number of patents initially increases, followed by a decrease. As shown in [Fig. 4], the number of patents registered for LCDs was the largest with 817 cases, and it is currently showing no further increase, as can be seen in [Fig. 3], indicating that the LCD technology has reached its maturity stage. 


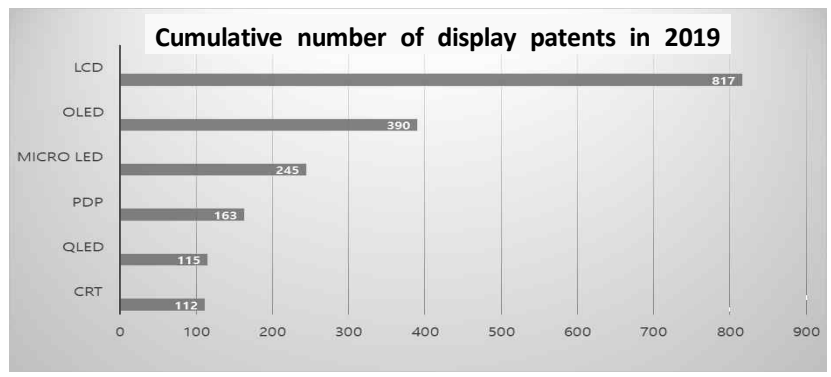

[Fig. 4] Cumulative Number of Display Patents in 2019[9]

\subsection{Position and Trend Analyses Using S-Curve}

The following diagrams are the S-curves of the CRT, LCD, PDP, OLED, QLED, and Micro LED technologies created based on the number of TV display patents. The position of each display technology and the technological trend were identified using the S-curves of the TV displays based on the number of patents. The technological trend suggests that among the six types of displays mentioned earlier, Micro LEDs have been leading the technological trend of TV displays with a continuously increasing number of patents and with the highest number of recent patents. These results are shown in the study below.

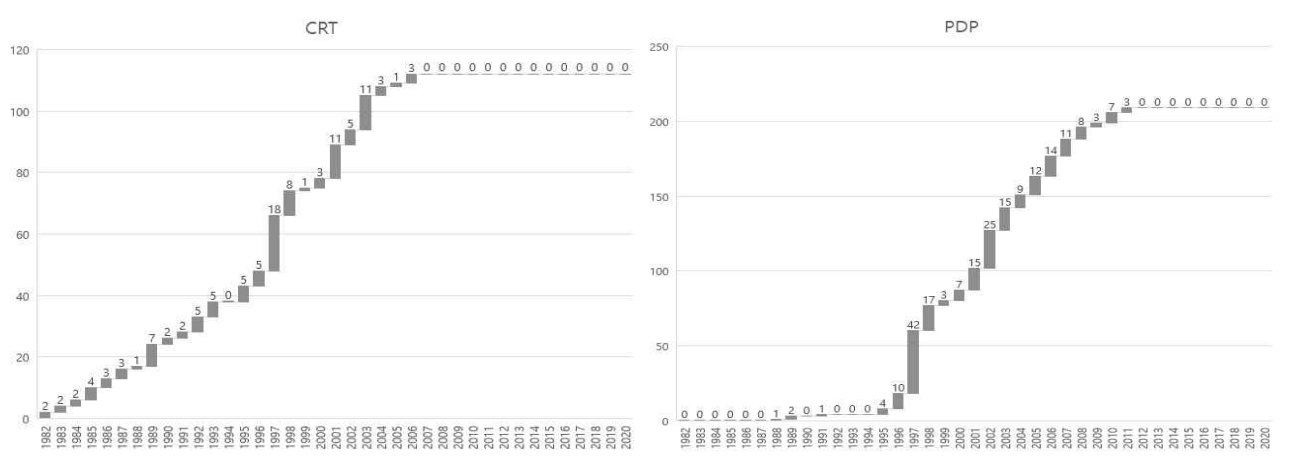

[Fig. 5] S-curves for the Numbers of CRTs and PDP Patents[9]

[Fig. 5] shows the S-curves created based on the number of CRT and PDP patents. Based on the cumulative number of patents suggested earlier, CRTs are in the decline stage, and no patents have been registered since 2007. In other words, with no patents presented at all in recent years, this can be regarded as a system that is no longer studied or developed and has become obsolete. The S-curve based on the number of patents for PDPs also shows that no 
patents have been registered since 2011. Therefore, CRTs and PDPs are currently in their technological decline.
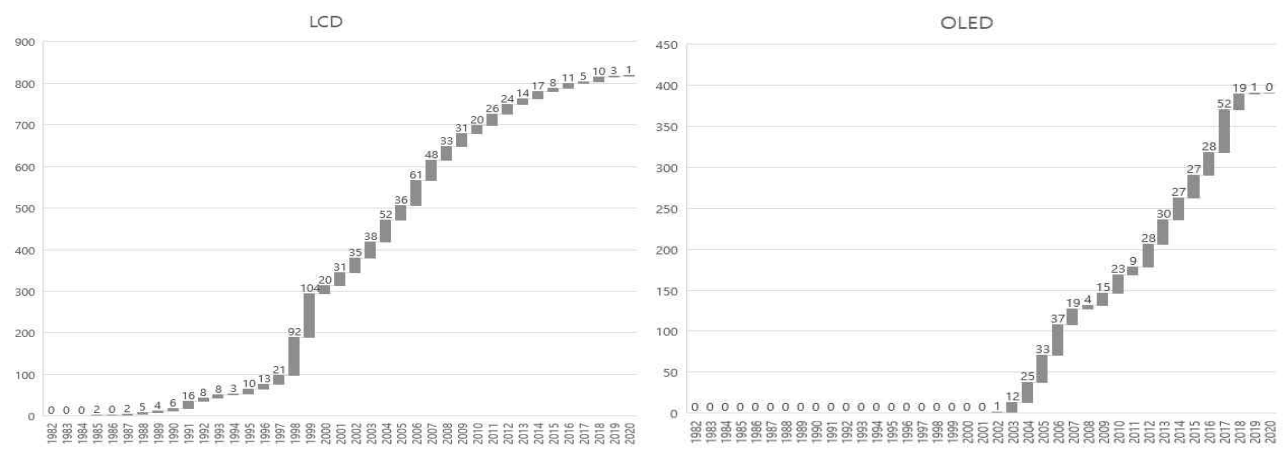

[Fig. 6] S-curves for the Numbers of LCD and OLED Patents[9]

[Fig. 6] shows the S-curves created based on the number of LCD and OLED patents. Unlike for CRTs and PDPs, patents are still being registered for LCDs. Nevertheless, the recent decrease in the number of new patents suggests that LCDs are in their maturity stage and no more technological improvements are being made. For OLEDs, the number of patents continuously increased during 2018, but as the number of patents has rapidly decreased since 2019, it is expected that they are located somewhere between the growth and maturity stages. OLED technology appears to be in its transition to the maturity stage.

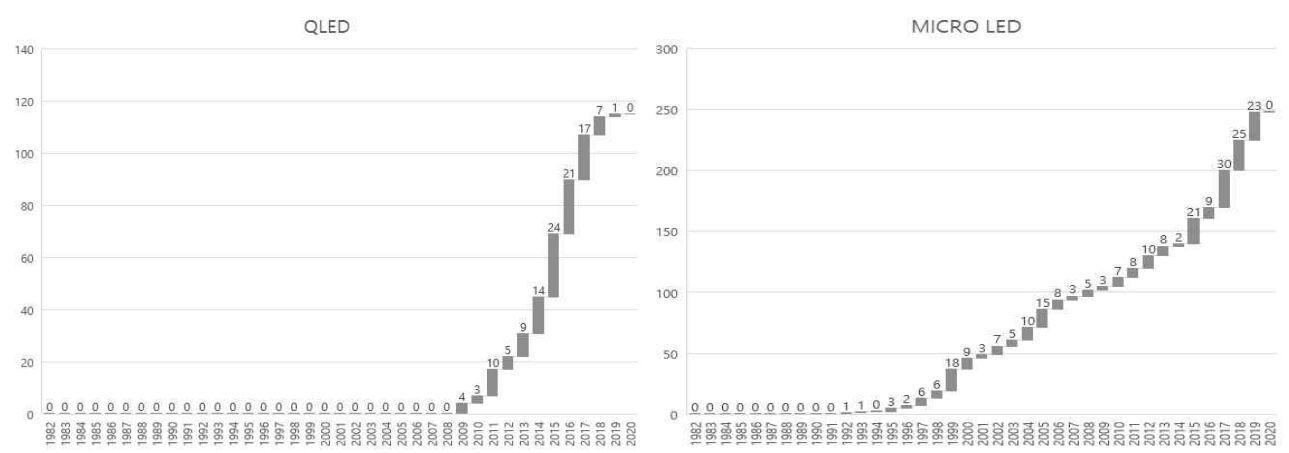

[Fig. 7] S-curves for the Numbers of QLED and Micro LED Patents[9]

[Fig. 7] shows the S-curves created based on the number of QLED and Micro LED patents. Although the number of QLED patents registered is insufficient for an evaluation owing to the short history of the technology since its first patent was registered in 2009, the relationship between OLEDs and QLEDs appears to follow a similar trend with the predominance of LCDs 
in competition with PDPs when considering the decreased number of patents since 2018. QLEDs seem to have entered the maturity stage in which the technology is longer progressing since its birth and growth. Meanwhile, the number of registered Micro LED patents has been constantly increasing since 1992, and the number of new patents for Micro LEDs is surpassing that of all other displays mentioned thus far. In other words, Micro LEDs are the future displays located between their birth and growth stages, and the technology are continuously being developed.

\subsection{S-Curve Analysis of Technological Levels Based on Various MPVs}

The S-curves for the MPVs of the display were analyzed in order of resolution, size (in inches), contrast ratio, viewing angle, image quality, power consumption, thickness, response speed, and price. S-curves were drawn through product research on 55-inch products from each year to analyze the technological trend and level based on the display type. Because it is difficult to draw an S-curve for the image quality through product research, the factors affecting the image quality, such as the number of pixels per inch (PPI), aperture ratio, and luminance were considered in the analyses. The power consumption was analyzed by diving the power consumption of 55-inch TVs according to the resolution.
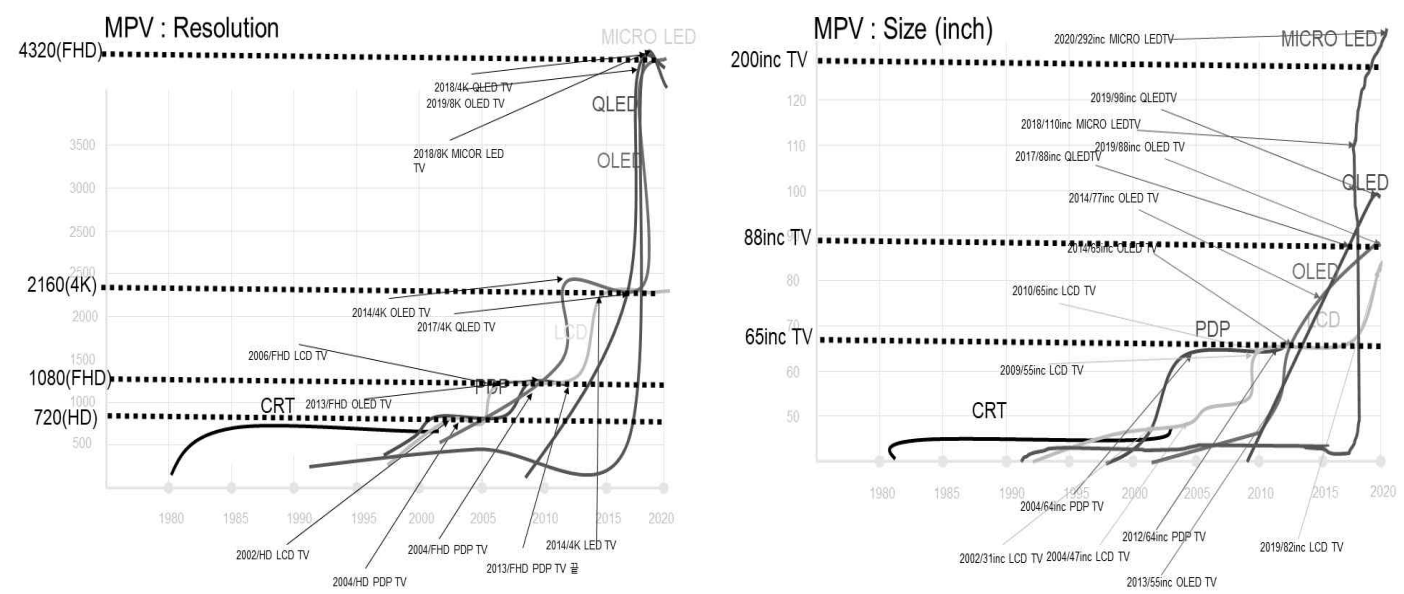

[Fig. 8] S-curves for Resolution and Size[10]

According to the S-curves for the resolution shown in [Fig. 8], CRT TVs entered the HD level in approximately 2001 after their first appearance during the 1980s. Since their introduction in 1996, PDP TVs reached the HD and FHD resolutions in 2004, and the technology remained 
at the level of FHD resolution until 2013 with no further improvements. LCD TVs were introduced in 1996, including PDPs, and the technology reached the HD level in 2002, the FHD level in 2006, and the $4 \mathrm{~K}$ level in 2014. They are still being developed at a $4 \mathrm{~K}$ resolution. OLED TVs were first released in FHD in 2013, the technology reached the 4K level in 2015, and the 8K level in 2019, and is still being developed at this level. QLED TVs were released in 2017 at the technological level of $4 \mathrm{~K}$, and have currently reached the $8 \mathrm{~K}$ level, similar to OLED TVs. Since the introduction of the prototypes of Micro LED-based TVs at CES2018, the technology has reached the level of $8 \mathrm{~K}$ as of 2018 and is still being developed. In other words, the S-curves for the resolution indicate that Micro LEDs, OLEDs, and QLEDs are currently being developed in $8 \mathrm{~K}$. The S-curves for the size (in inches) shown in [Fig. 8] indicate that, after their introduction in the 1980s, CRT TVs reached their technological height at under 50 inches by 2001. Since their introduction during the late 1990s, PDP TVs had reached the technological level of 55 inches in 2004 and were being developed at the 64-inch level in 2012. LCD TVs were released at a 31-inch size in 2002, and the technology developed to the 55-inch level by 2009 and the 82-inch level by 2019, and their development is still in progress. The first 55-inch OLED TVs were released in 2013, the technology reached the 88-inch level in 2019, and their development is still ongoing. QLED TV technology reached the 88-inch level in 2017 and the 98-inch level in 2019, and the development of this technology is continuing. Prototype Micro LED TVs with 110-inch displays were introduced at CES2018, and at CES2020, prototypes with 292-inch displays were introduced, boasting the overwhelming size of Micro LED displays compared to the other display types[11].

[Fig. 9] shows the S-curves comparing each display based on the MPVs set to the contrast ratio and viewing angle, respectively. The S-curves for contrast ratios suggest that CRT TVs have an extremely low contrast ratio of below 10,000:1. PDP TVs reached a 10,000:1 contrast ratio in 2006. With the improvement of technology, the contrast ratio was enhanced to 1,000,000:1 in 2007, 2,000,000:1 in 2009, and 10,000,000:1 in 2012. The LCD TVs reached the technological level of 1,000,000:1 in 2006. OLED and Micro LED TVs have an almost infinite contrast ratio. Although QLEDs also have a high level of technology, it is still not even close to the overwhelming level of contrast ratio technology found in Micro LED and OLED TVs. In the viewing angle S-curves, the viewing angle of all TVs except CRT TVs are at a similar level of technology at 178 degrees. In general, IPS panels are used in the production of TVs[12], and an IPS has vertical and horizontal viewing angles of 178 degrees. Therefore, the S-curves show similar viewing angles at 178 degrees for all TVs except CRT TVs. 

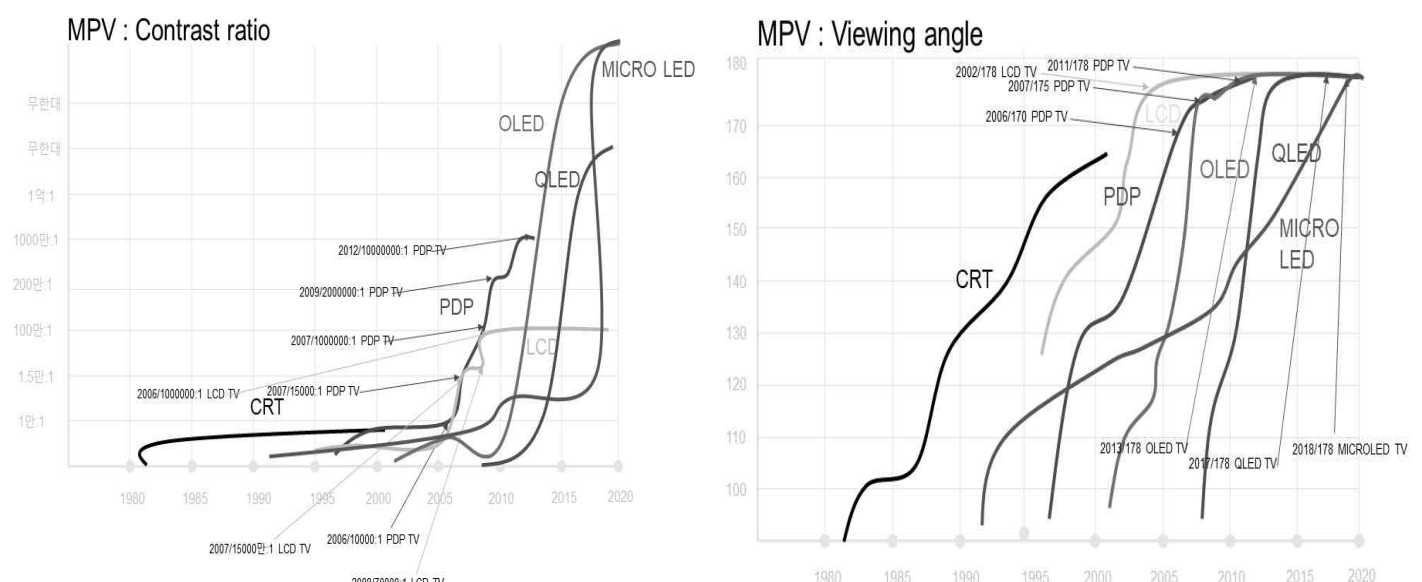

[Fig. 9] S-curves for Contrast Ratio and Viewing Angle[10]
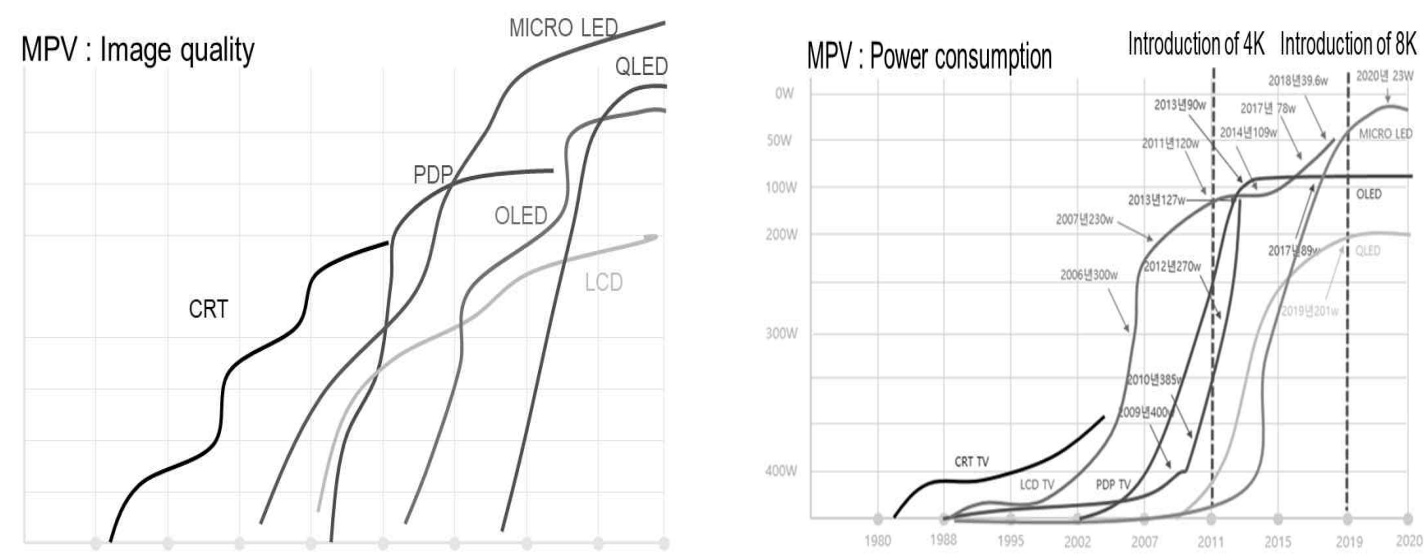

[Fig. 10] S-curves for Image Quality and Power Consumption[10]

In [Fig. 10], the MPV (image quality) is determined by a variety of complex factors in the TV; for example, the brightness of the LED and the image quality of the TV depend on various complex factors such as the clarity of the display, aperture ratio, PPI, and the light emission method applied. Therefore, the S-curves for the MPV (image quality) were briefly created through predictions based on published papers and articles. From the research based on such predictions, the highest technological image quality was found in Micro LEDs, followed by QLEDs, OLEDs, LCDs, PDPs, and CRTs. In terms of the next MPV (power consumption), CRT TVs remained at a technological level of $300 \mathrm{~W}$ or more from 1983 to 2001. PDP TVs reached a technological level of $400 \mathrm{~W}$ in 2009, $385 \mathrm{~W}$ in 2010, $270 \mathrm{~W}$ in 2012, and $127 \mathrm{~W}$ in 2013, with no more products released at a higher level of technology. LCD TVs demonstrated 
an unimpressive level of power consumption until 2005, but since reaching $300 \mathrm{~W}$ in 2006, rose to the levels of $230 \mathrm{~W}$ in 2007, $120 \mathrm{~W}$ in 2011, $109 \mathrm{~W}$ in 2014, $78 \mathrm{~W}$ in 2017, and $39.6 \mathrm{~W}$ in 2018 with continuing development. Although the technological level of OLED TVs before 2013 could not be evaluated because the TVs had not yet been commercialized, the technology reached the level of $90 \mathrm{~W}$ in 2013, and their development is still ongoing. QLED TVs were first released in 2017 at a power level of $201 \mathrm{~W}$, and their level of technology in this regard is still quite low despite ongoing development. Finally, since the introduction of the first Micro LED TV prototypes at CES2018, the technology has reached a level of $23 \mathrm{~W}$ in 2020 with continuing development. In other words, the highest level of technology in terms of power consumption was found to be in Micro LEDs, followed by LCDs, OLEDs, PDPs, QLEDs, and CRTs. The S-curve analysis based on the product research revealed that the technological level of QLED TVs was lower than expected. The technological level of LCD TVs appeared to be higher than that of OLEDs, which consume more power.
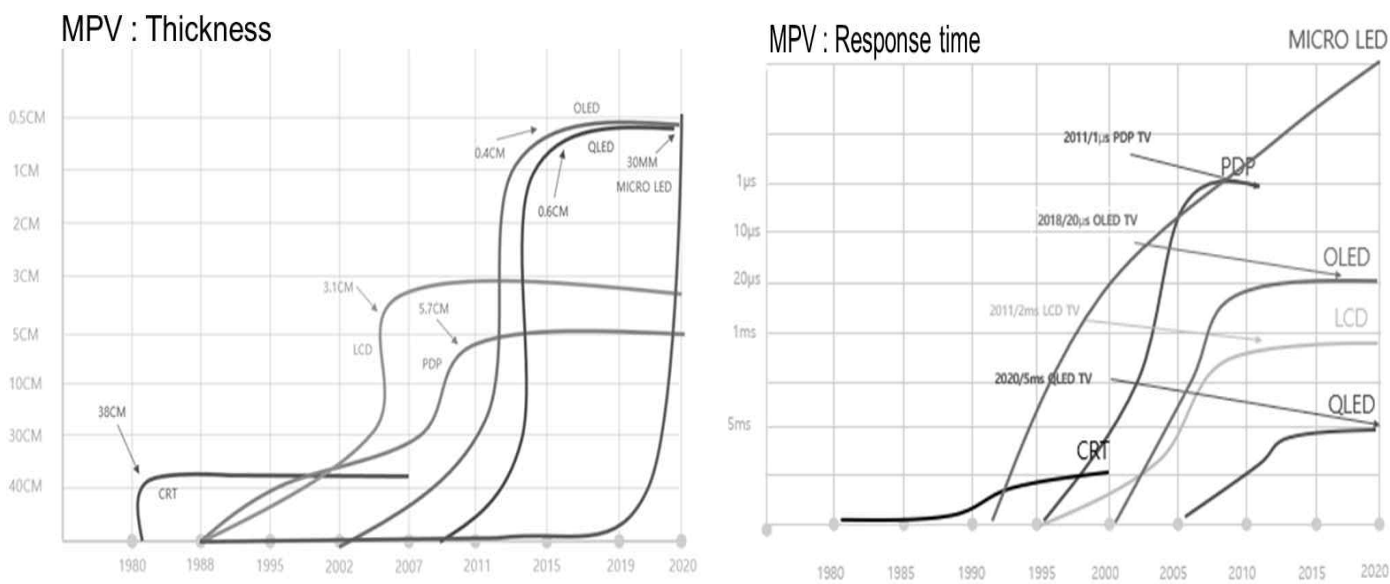

[Fig. 11] S-curves for Thickness and Response Time[10]

[Fig. 11] shows the S-curves in terms of the thickness of the display and response time. According to the S-curves for thickness, CRT TVs have been developed to a technological level of $38 \mathrm{~cm}$ as of 2007 since the 1980s. Since their birth in 1990, PDP TVs reached a technological level of $5.7 \mathrm{~cm}$ during the 2000s with no further improvement in this technology. LCD TVs reached the technological level of $3.1 \mathrm{~cm}$ during the 2000s and have maintained this level to date. Since their birth during the 2000s, OLED TVs reached a technological level of $0.4 \mathrm{~cm}$ after 2015. QLED TVs were released in the mid-2000s and reached a technological level of 0.6 $\mathrm{cm}$ after 2015. Micro LEDs demonstrated a technological advancement to $30 \mathrm{~mm}$ in 2020, and 
their development is still ongoing. Therefore, to date, the highest technological level in terms of the thickness of the display was found in Micro LEDs, followed by OLEDs and QLEDs. As TV displays evolve from QLEDs and OLEDs into Micro LEDs, TV displays are becoming thinner with a simplification of the TV transmission system. A shorter response time indicates a faster response of the pixels, enabling a clearer view of moving images. According to the S-curve above, the response time of CRT TVs appears to be extremely long. PDP TVs reached a technological level of 1 us in 2011. LCD TVs reached a technological level of $2 \mathrm{~ms}$ in 2011. OLED TVs reached a technological level of $20 \mu \mathrm{s}$ in 2018. QLED TVs reached a technological level of $5 \mathrm{~ms}$ in 2020. Although Micro LED TVs have not been commercialized yet, they appear to have a level of technology surpassing that of all other preceding TVs with an extremely fast response time based on the specifications announced at CES2020[13]. Therefore, the technological level is the highest in Micro LEDs, followed by PDPs, and OLEDs.

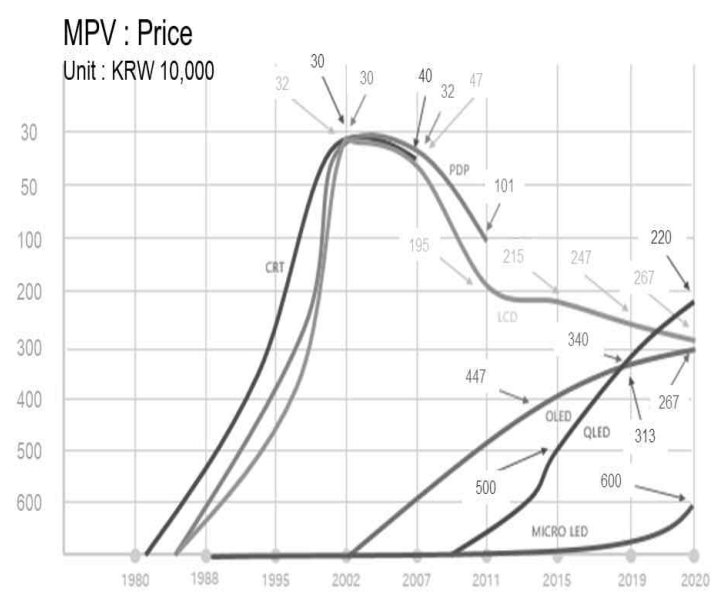

[Fig. 12] S-curves for Price[10]

[Fig. 12] shows the S-curves in terms of price. According to the S-curve, the price for CRT TVs was approximately KRW 300,000 from the late 1990s to the early 2000s. The PDP TVs cost approximately KRW 300,000 during the early 2000s, and KRW 1,010,000 in 2011 based on the S-curve analysis. The S-curve of LCD TVs show increase in prices from KRW 320,000 during the early 2000s to KRW 470,000 in 2007, KRW 1,960,000 in 2011, KRW 2,050,000 in 2015, KRW 2,470,000 in 2019, and KRW 2,670,000 in 2020. The S-curve of OLED TVs show prices of KRW $4,470,000$ in 2015, KRW 3,400,000 in 2019, and KRW 2,760,000 in 2020. The QLED TV prices were KRW 5,000,000 in 2015, KRW 3,130,000 in 2019, and KRW 2,200,000 in 2020. Micro LED TVs are yet to be commercialized. In terms of price, as presented at CES2020, they are still a 
long way from commercialization owing to the high-end and high-cost processing required and a high-cost of KRW 6,000,000 for basic materials alone[14]. Therefore, the price of the Micro LEDs was the highest, followed by OLEDs and QLEDs. Micro LEDs appear to be the most efficient products in all aspects with an extremely high price. However, the price can be reduced by mass production through technological development[15] and it seems to be a factor that can be overcome.

\subsection{Results of S-Curve Analysis for Various MPVs}

[Table 2] Ranks of Technological Level based on MPV S-curves

\begin{tabular}{|c|c|c|c|c|c|c|}
\hline & CRT & PDP & LCD & OLED & QLED & Micro LED \\
\hline Resolution & 6 & 5 & 4 & 1 & 1 & 1 \\
\hline $\begin{array}{l}\text { Contrast } \\
\text { ratio }\end{array}$ & 6 & 4 & 5 & 3 & 1 & 1 \\
\hline $\begin{array}{l}\text { Image } \\
\text { quality }\end{array}$ & 6 & 4 & 5 & 2 & 3 & 1 \\
\hline $\begin{array}{l}\text { Viewing } \\
\text { angle }\end{array}$ & 6 & 1 & 1 & 1 & 1 & 1 \\
\hline Size (inches) & 6 & 5 & 4 & 2 & 3 & 1 \\
\hline $\begin{array}{c}\text { Power } \\
\text { consumption }\end{array}$ & 6 & 4 & 3 & 5 & 2 & 1 \\
\hline Price & 1 & 2 & 3 & 4 & 5 & 6 \\
\hline $\begin{array}{l}\text { Response } \\
\text { time }\end{array}$ & 6 & 2 & 3 & 4 & 3 & 1 \\
\hline Thickness & 6 & 5 & 4 & 3 & 2 & 1 \\
\hline Total rank & 4.78 (6th) & 3.56 (4th) & 3.56 (4th) & 2.78 (3rd) & 2.3 (2nd) & 1.56 (1st) \\
\hline
\end{tabular}

[Table 2] shows the rank of each item based on the S-curve analysis of the MPVs. According to [Table 2], the total rank of the technical level is in order of Micro LEDs, OLEDs, QLEDs, LCDs, PDPs, and CRTs, from the highest to lowest. Although Micro LEDs have similar or superior MPVs compared to all other TV displays, the problem of price, which is an important aspect in terms of consumer needs, has yet to be resolved. However, OLED TVs were extremely expensive during their early stage, but production costs have been reduced to an affordable level owing to mass production through technological development[16]. The price for Micro LEDs is incredibly high as of now, but they have been evaluated as the products that can be commercialized with the continued development of production technology[15] 


\section{Conclusion}

In this study, a technological system life cycle analysis method (S-curve), the basic step for the analysis of future technology prediction using the trend of technological system evolution, was applied to the development of TV displays. Based on the results, it was found that micro LEDs have been predicted to be developed into displays for future TVs by S-curves changes through patent analysis and analyzing the trends and technological positions. In addition, the technologies for the future TV industry were predicted by evaluating the level of each display technology with a variety of MPV S-curves. Micro LED TV technology was predicted to be the next-generation technology that will lead the future TV industry with a superior functionality despite a high price[9][10][15].

However, there is a limit to the future prediction method using S-curves only. Although S-curves can be used to analyze the development stage of technology, it does consider various external factors caused by environmental changes. It is necessary to analyze not only the technological aspects but also the social and cultural factors that influence the product[4][5]. Therefore, the development of a comprehensive future prediction methodology incorporated with sociocultural trends is required.

\section{Acknowledgement}

This research was supported by Basic Science Research Program through the National Research Foundation of Korea (NRF) funded by the Ministry of Education (2018R1D1A1B07049244)

\section{References}

[1] Nam Doo, Methodology of strategic future forecasting, The Korea Association for Future Studies, (2014)

[2] Seok-Woon Jung, Se-Il Nam, Seok Hong, Chang-Hee Han, Future technology foresight for an enterprise: Methodology and case, Journal of Society for e-Business Studies, (2006), Vol.11, No.1, pp.26-89.

[3] Genrich Altshulle, Creativity as an exact science, Translated by Seong-Kyun Park, Korea: Intervision, (2006)

[4] Boris Zlotin, Alla Zusman, Frank Halfell, TRIZ to invent your future utilizing directed evolution methodology, Procedia Engineering, (2011), Vol.9, pp.126-134.

[5] Yong-Wond Song, Kyung-Mo Kim, Seong-Hwan Kim, Theory of inventive problem solving, Korea: Korea 
Standards Association Media, (2017)

Asia-pacific Journal of Convergent Research Interchange

Vol.6, No.10, October 31 (2020), pp.147-163 http://dx.doi.org/10.47116/apjcri.2020.10.12

[6] Byung-Moo Heo, A study on the dominant design factors and determinants in the product evolution of TVs, Kookmin University, Ph.D dissertation, (2016)

[7] https://namu.wiki/w/\%EC\%9E\%A5\%EC\%8B\%9C\%EA\%B0\%84\%20\%EC\%9D\%8C\%EB\%B0\%98, Aug 25 (2020)

[8] https://www.mk.co.kr/news/business/view/2020/04/442352/, Apr 29 (2020)

[9] http://www.kipris.or.kr/khome/main.jsp, Jan 14 (2020)

[10] http://www.danawa.com/?src=adwords\&kw=GA0000020\&gclid=EAIaIQobChMIoo7Wr-S96gIVVLaWCh1KIg REAAYASAAEgKZ_vD_BwE, Jan 19 (2020)

[11] http://www.inews24.com/view/1234847, Jan 10 (2020)

[12] https://social.lge.co.kr/product/lg_nanocell_0829/, Aug 29 (2017)

[13] https://www.etnews.com/20200131000109?m=1, Feb 2 (2020)

[14] https://www.yna.co.kr/view/AKR20200118034000003, Jan 19 (2020)

[15] In-Ho Hwang, Next-generation display industry expected to grow rapidly in micro LED displays, NICE Information Service-Korea IR Council, (2019)

[16] https://cmobile.g-enews.com/view.php?ud=2020041411075536042f6424d93c_1\&ssk=favorset\&md=2020041415 0014_R, Apr 15 (2020) 